\title{
An Improved Pediatric Weight Estimation Strategy
}

Open Access

\author{
Susan M Abdel-Rahman* and Anna L Ridge
}

Division of Clinical Pharmacology and Medical Toxicology, The Children's Mercy Hospitals and Clinics, 2401 Gillham Rd., Suite 0411, Kansas City, MO 64108, Missouri

\begin{abstract}
There exist a number of pediatric health care settings wherein time and/or resource constraints do not permit care providers to reliably assess children's weight. This study describes the development and validation of a pediatric weight estimation strategy that addresses the limitations of currently available weight estimation methods. Demographic and anthropometric data on children 2 months to 16 years of age were extracted from the National Health and Nutrition Examination Survey. Datasets were randomly assigned into a method development set $(\mathrm{n}=17,328)$ and a method validation set $(n=1,938)$. Humeral length and mid-upper arm circumference were used to develop a weight estimation method. The predictive performance of this method was evaluated and compared with the performance of 13 previously published weight estimation methods. We also developed a measurement device that performs both measurements simultaneously requiring simple addition and no external references to arrive at the weight estimate. The method developed in this study (Mercy method) outperformed the 13 other published methods when evaluated by goodness-of-fit $(\mathrm{r}=0.98$ vs. 0.69 to 0.87 ; slope $=0.97$ vs. 0.43 to 0.96 ; intercept 0.9 vs. 3.1 to 11.8 ), mean error ( $-0.40 \mathrm{~kg}$ vs. -10.88 to 2.23 ), mean percentage error (- $-0.46 \%$ vs. -16.84 to 3.51$)$, root mean square error (3.65 kg vs. 3.42 to 16.96$)$ and percentage of children in agreement within $10 \%$ of actual weight ( $79 \%$ vs. 17.8 to 45.3 ). The Mercy method represents a significant improvement over existing age-based, length-based and habitus-based weight estimation strategies.
\end{abstract}

Keywords: Weight estimation, Mercy TAPE, pediatric, emergency, global health.

\section{INTRODUCTION}

In 'real-world' health care settings there exist a number of circumstances where the weight of a child is desirable or even necessary but unavailable. The most conspicuous of these settings can be found in developing countries where many medical clinics lack suitable scales to obtain accurate infant and child weights [1,2]. Though resource restrictions are less of an issue in developed countries, there still exist scenarios where weight assessment is problematic. Accurate estimates of a child's weight are rarely available during emergency or trauma situations, and in some in-patient settings obtaining an accurate patient weight can be impaired by the presence of external hoses, tubing and/or other medical equipment. Irrespective of the environment, the challenge that these settings present is the same; namely, the provision of age-appropriate therapy with weight-based drug doses which remains the most accurate approach to delivering medicines in children.

Apart from parental recall which can vary in accuracy [35], the most commonly used strategies for estimating weight rely on the child's age, length, or a combination of the two parameters. While simple and easy to integrate into clinical practice, age-based strategies fail to account for the extremes of body composition and stature that are observed in children

\footnotetext{
*Address correspondence to this author at the Division of Clinical Pharmacology and Medical Toxicology, The Children's Mercy Hospitals and Clinics, 2401 Gillham Rd., Suite 0411, Kansas City, MO 64108, Missouri; Tel: 816-234-3059; Fax: 816-855-1759;

E-mail: srahman@cmh.edu
}

of the same age. Similarly, length-based strategies do not take into consideration that two children of the same height may demonstrate markedly discrepant weights based on underlying nutritional status (e.g. malnourished, underweight, overweight, obese) [6]. Consequently, many of the currently available weight estimation strategies perform well in only a small subset of children [7]. Some weight estimation strategies present additional complexities for the end-user including; the need for subjective assessments of habitus, the requirement to solve exponential equations, the call for multiple formulae by age bracket, and the reliance on one or more reference charts [8-12].

Despite the relative abundance of weight estimation strategies that exist, there remains a critical need for methods that are accurate across a wide range of pediatric ages, weights, lengths, nationalities and body compositions [7]. We describe the development and validation of a weight estimation method that incorporates surrogates for both stature and body habitus, requires no subjective assessment and performs robustly independently of age and length over a broad range of weights. We also describe the development of a simple and inexpensive device that can perform the two measurements simultaneously and report the predicted weight directly from the device as opposed to consulting a separate table or chart.

\section{MATERIALS AND METHODS}

\section{Method Development}

The National Health and Nutrition Examination Survey (NHANES) datasets corresponding to the years 1999-2000, 
a.

\begin{tabular}{|c|c|c|c|}
\hline $\begin{array}{l}\text { Humeral } \\
\text { Length } \\
\text { (cm) }\end{array}$ & $\begin{array}{c}\text { Partial } \\
\text { Weight A } \\
\text { (kg) }\end{array}$ & $\begin{array}{l}\text { Mid-upper arm } \\
\text { Circumference } \\
\text { (cm) }\end{array}$ & $\begin{array}{c}\text { Partial } \\
\text { Weight B } \\
\text { (kg) }\end{array}$ \\
\hline 9 & 0.5 & 10 & 2.8 \\
\hline 10 & 0.7 & 11 & 3.8 \\
\hline 11 & 0.9 & 12 & 4.6 \\
\hline 12 & 1.5 & 13 & 4.9 \\
\hline 13 & 2.0 & 14 & 5.3 \\
\hline 14 & 2.8 & 15 & 5.9 \\
\hline 15 & 3.4 & 16 & 6.5 \\
\hline 16 & 4.2 & 17 & 7.4 \\
\hline 17 & 5.0 & 18 & 8.0 \\
\hline 18 & 6.1 & 19 & 9.4 \\
\hline 19 & 7.2 & 20 & 10.9 \\
\hline 20 & 8.1 & 21 & 12.4 \\
\hline 21 & 9.1 & 22 & 14.3 \\
\hline 22 & 10.4 & 23 & 16.5 \\
\hline 23 & 11.4 & 24 & 18.0 \\
\hline 24 & 12.6 & 25 & 20.5 \\
\hline 25 & 13.7 & 26 & 23.4 \\
\hline 26 & 14.7 & 27 & 25.5 \\
\hline 27 & 16.6 & 28 & 27.8 \\
\hline 28 & 18.3 & 29 & 30.5 \\
\hline 29 & 19.6 & 30 & 33.3 \\
\hline 30 & 21.4 & 31 & 36.3 \\
\hline 31 & 23.7 & 32 & 39.6 \\
\hline 32 & 25.5 & 33 & 44.8 \\
\hline 33 & 27.3 & 34 & 46.5 \\
\hline 34 & 29.2 & 35 & 50.2 \\
\hline 35 & 31.0 & 36 & 53.2 \\
\hline 36 & 33.5 & 37 & 55.7 \\
\hline 37 & 34.5 & 38 & 60.3 \\
\hline 38 & 36.5 & 39 & 61.1 \\
\hline 39 & 38.2 & 40 & 67.0 \\
\hline
\end{tabular}

b.
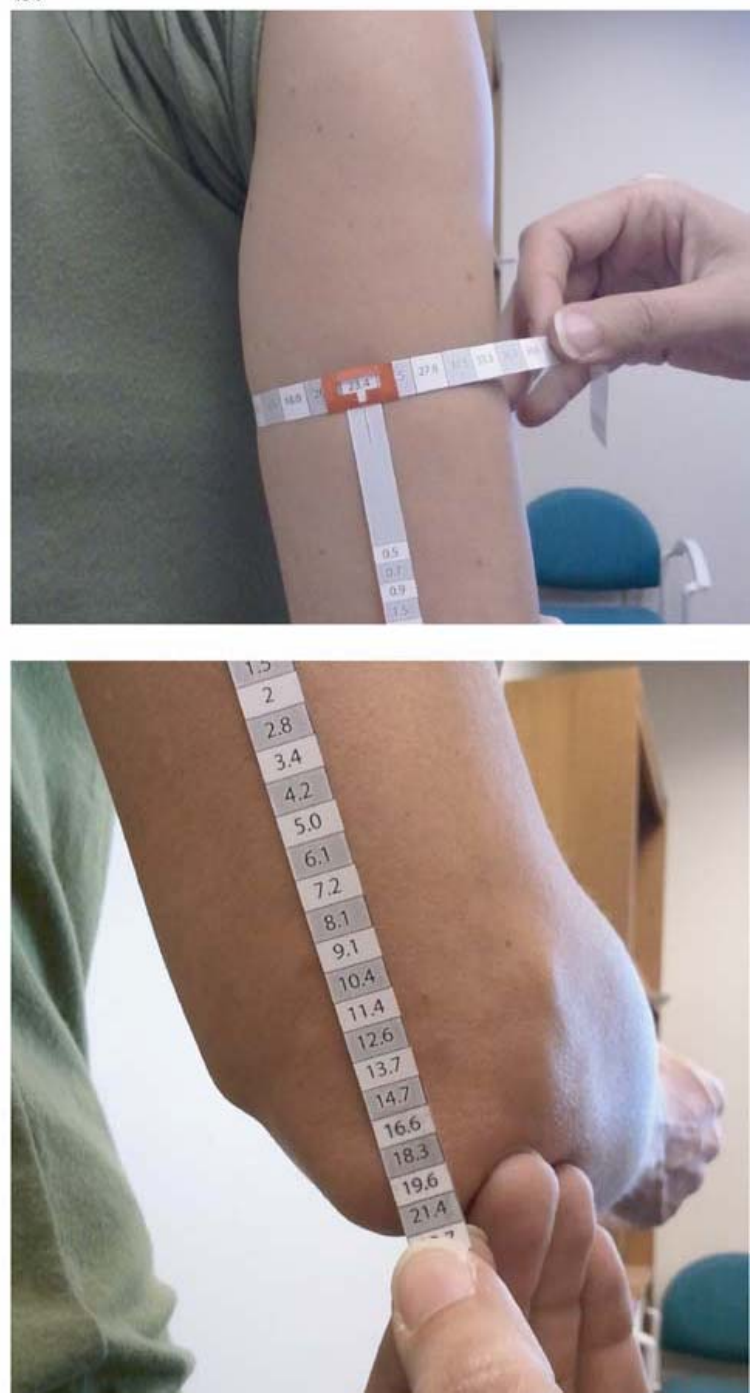

Fig. (1). The Mercy method and the Mercy TAPE (a). Humeral length and mid-upper arm circumference bins with their corresponding fractional weight values. A weight estimate is generated for each individual by the simple addition of the MUAC and HL fractional bin value that corresponds to that individuals measurements (b). The Mercy TAPE being used to measure MUAC and HHL simultaneously.

2001-2002, 2003-2004, 2005-2006 and 2007-2008 were downloaded from the CDC website [13] under a study that was reviewed and approved by the Institutional Review Board at Children's Mercy Hospital. The subset of data constituting all children aged 0 to 16 years were extracted into a separate database. Demographic and anthropometric variables of interest included age, gender, height, length, weight, middle-upper arm circumference (MUAC), and humeral length (HL). Incomplete datasets and those missing the relevant variables were excluded. A random number generator was used to partition the datasets into the "method development" and the "method validation" sets with approximately $90 \%$ and $10 \%$ of the datasets comprising the former and later groups, respectively.

The relationship between total body length and HL was explored prior to method development to confirm the utility of long bone length serving as a surrogate for total body length. Similarly, the relationships between HL or MUAC (defined as continuous variables) and weight were examined to confirm their utility as variables in an equation describing weight. Length and habitus measurements were collapsed into $1.0 \mathrm{~cm}$ bins creating a finite number of discrete variables by rounding MUAC and HL up or down to their nearest 1.0 $\mathrm{cm}$ increment. The median population weight from the method development dataset was calculated for each MUAC-HL bin pair. A statistical weighting value was assigned to each bin pair depending on the absolute number of individuals in the population that comprised the median weight of that bin pair. Additionally, an inverse weighting value was assigned to each bin pair based on the median age of the individuals that comprised the corresponding MAUCHL bin. Two-dimensional least-squares regression was undertaken to identify a fractional weight assignment that would minimize the goodness-of-fit criteria (e.g. residuals, sum-of-squares, etc) for each HL bin across all MUAC bins, for each MUAC bin across all HL bins and for all MUAC and HL bins taken together. A weight estimate is generated for each individual by the simple addition of the MUAC and HL fractional bin values that correspond to that individuals measurements (Fig. 1a). 
Table 1. Demographic Variables of Children in the Method Development and Validation Sets

\begin{tabular}{|c|c|c|}
\hline Parameter & Development Set & Validation Set \\
\hline \hline size & 17,328 & 7.938 \\
\hline age $(\mathrm{yr})$ & $7.9 \pm 5.3$ & $36.6 \pm 24.8$ \\
\hline weight $(\mathrm{kg})$ & $35.9 \pm 24.2$ & $137.9 \pm 27.1$ \\
\hline height $(\mathrm{cm})$ & $137.8 \pm 26.7$ & $126.9 \pm 34.5$ \\
\hline length $(\mathrm{cm})$ & $126.8 \pm 34.3$ & $26.9 \pm 8.1$ \\
\hline HL $(\mathrm{cm})$ & $26.9 \pm 8.1$ & $21.8 \pm 6.3$ \\
\hline MUAC $(\mathrm{cm})$ & $21.7 \pm 6.2$ & \\
\hline
\end{tabular}

\section{Method Validation and Device Design}

The remaining $10 \%$ of data were used as an internal validation set. The MUAC and HL of each child was rounded up or down to the nearest $1.0 \mathrm{~cm}$ bin (e.g. HL of 19.2 is rounded down to 19 , MUAC of 32.7 rounded up to 33). The corresponding fractional weight for each variable was identified and summed to generate a weight estimate for that child. The age and/or length of each child was also used to generate a predicted weight using 13 other previously published weight estimation strategies. These included methods developed and/or published by Advanced Pediatric Life Support (APLS), the Australian Resuscitation Council (ARC), Argall, Best Guess, Broselow, Cattermole, Leffler, Luscombe and Owens, Nelson, Shann, Theron, Traub-Johnson, TraubKichen [3, 9-11, 14-22]. For each estimation method, no weight was estimated if the child's age and/or height fell outside of the bounds established for that method (e.g. weight would not be estimated using; the Broselow method for a $158 \mathrm{~cm}$ child, the Theron method for a 6 month old infant, etc). Initial model development was performed with MUAC and HL; however, model development was then refined using half-humeral length (HHL) for development of the device. This change was made because MUAC is determined at the midpoint of the humerus and the incorporation of a half-humeral measurement into the model would permit the construction of a simple tool that can simultaneously measure both variables at once. Validation was repeated using MUAC and HHL rounded to the nearest 1.0 and $0.5 \mathrm{~cm}$, respectively to confirm that the model returned identical performance statistics to the MUAC-HL model. Once confirmed, a 2-dimensional image was constructed consisting of adjacent perpendicular measuring strips partitioned to correspond to the respective MUAC and HHL bins. The image is cut out and the product folded in a specific manner to generate a 3-dimensional measuring device which permits the assessment of MUAC and HHL at one time. Importantly, the device does not register a centimeter length reading for MUAC and HHL but rather directly displays the fractional weight corresponding to each variable such that the two values are simply added together with no additional conversion or external reference required. The device is depicted in Fig. (1b). Alternatively, the tool can be constructed as a singular strip with bins for MUAC mapped on one side and bins for HL mapped on the other. This version would require two independent measurement steps followed by addition as is done with the 3D unit. For populations with done with the 3D unit. For populations with less variability MUAC and HHL bins can be truncated with no introduction of error.

\section{Statistical Analysis}

The predicted weight of each child was plotted against their actual weight to evaluate the predictive performance of the mathematical model developed in this study (the "Mercy" method) and the previously published methods described above. Linear regression was used to evaluate the relationship between the actual and predicted weights (i.e. the validity of the method) by calculating the slope, the $95 \%$ confidence interval (CI) for the slope, the intercept and the correlation coefficient. Mean error (ME) was calculated by taking the difference of the predicted and actual weights. Mean percentage error (MPE) between predicted and actual weight was calculated by dividing by the actual weight into the ME and multiplying by 100 . Fortuitous cancellation of errors with different signs was examined by computing the root mean square error (RMSE) calculated by taking the square root of the average squared error. Analysis of variance on the error estimates was used to determine the statistical significance between models. The percent agreement between estimated weight and actual weight is also reported. The predictive performance of the Mercy method was also evaluated across demographic subgroups (i.e. race/ethnicity and gender). Finally, Bland-Altman plots were constructed to evaluate the agreement between each of the weight estimation methods and observed weight. All mathematical and statistical analyses were performed with Microsoft Excel 2003 and SPSS v 12.0.

\section{Results}

Only children greater than 2 months of age in the NHANES database had humeral length and MUAC recorded. When children under the age of 2 months were removed, along with the remaining incomplete data sets, a total of 19,266 pediatric datasets were available. Of these, 17,328 constituted the method development set and the remaining 1,938 comprised the method validation set. Selected demographic and anthropometric parameters for the children in each data set are provided in Table $\mathbf{1}$. The overall performance of the Mercy method is illustrated in Fig. (2) and the regression parameter estimates for our method compared with other weight-estimation strategies are provided in Table 2.

Figs. (3 and 4) provide a graphical illustration of the precision and bias for each method. When examined for bias 


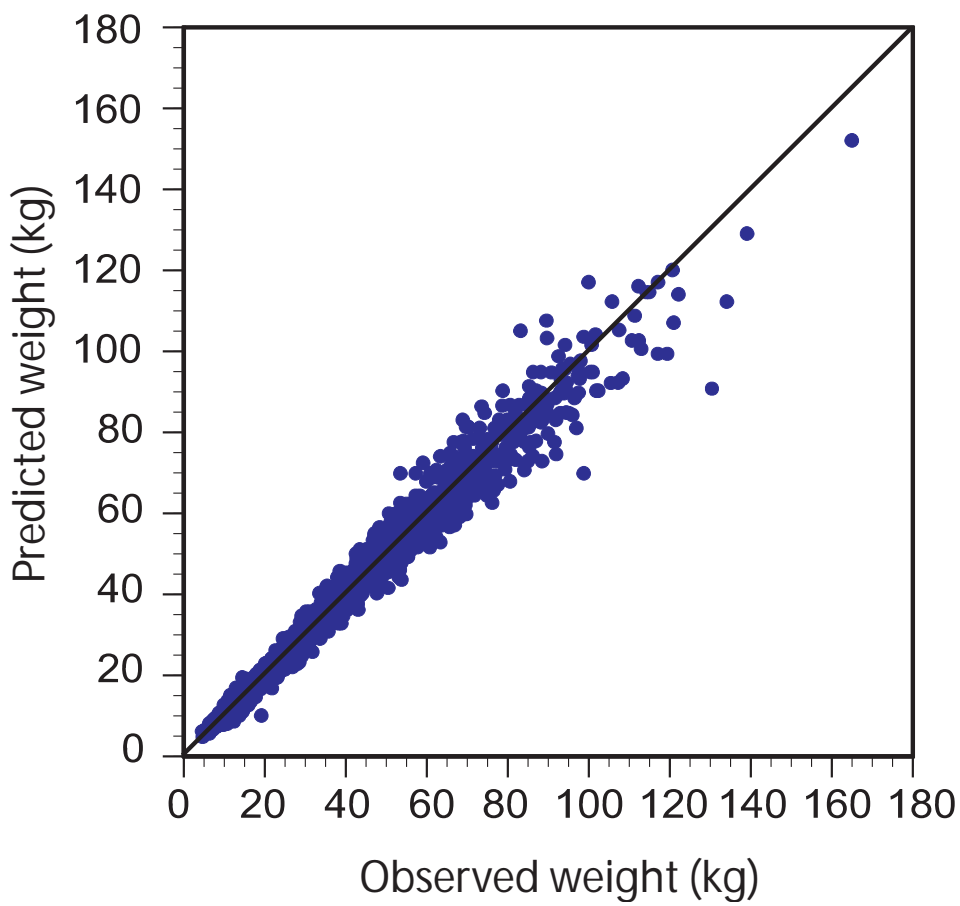

Fig. (2). Overall performance of the Mercy method. Actual versus predicted weights derived using the Mercy method for the 1,938 children in the validation data set.

Table 2. Weight Estimation Methods Evaluated in this Study Provided with the Goodness-of-fit Criteria Between Actual and Predicted Weights

\begin{tabular}{|c|c|c|c|c|c|c|c|c|}
\hline Method & $\begin{array}{c}\text { Basis for } \\
\text { Weight }\end{array}$ & $\begin{array}{c}\text { Lower Age } \\
\text { Limit }\end{array}$ & $\begin{array}{c}\text { Upper Age } \\
\text { Limit }\end{array}$ & $\begin{array}{c}\text { Length } \\
\text { Restrictions }\end{array}$ & $\%$ Predicted $^{\mathbf{a}}$ & Slope $[95 \%$ CI $]$ & Intercept & $\mathbf{r}^{2}$ \\
\hline Mercy method & MUAC \& HL & $2 \mathrm{mo}$ & $16 \mathrm{yr}$ & none & 100 & $0.965[0.958,0.971]$ & 0.9 & 0.98 \\
\hline APLS & age & $1 \mathrm{yr}$ & $10 \mathrm{yr}$ & none & 52.3 & $0.425[0.407,0.443]$ & 8.4 & 0.69 \\
\hline $\mathrm{ARC}$ & age & $1 \mathrm{yr}$ & none & none & 90.4 & $0.508[0.494,0.523]$ & 9.8 & 0.74 \\
\hline Argall formula & age & $1 \mathrm{yr}$ & $10 \mathrm{yr}$ & none & 52.3 & $0.638[0.611,0.664]$ & 6.6 & 0.69 \\
\hline Best Guess & age & none & $14 \mathrm{yr}$ & none & 80.6 & $0.666[0.647,0.684]$ & 8.0 & 0.76 \\
\hline Broselow & TBL & none & none & $46-143 \mathrm{~cm}$ & 62.6 & $0.727[0.710,0.744]$ & 3.8 & 0.86 \\
\hline Cattermole & MUAC & 6 & 11 & none & 27.3 & $0.866[0.837,0.895]$ & 5.3 & 0.87 \\
\hline Leffler & age & none & $10 \mathrm{yr}$ & none & 61.9 & $0.513[0.495,0.531]$ & 7.5 & 0.72 \\
\hline Luscombe \& Owens & age & $1 \mathrm{yr}$ & $10 \mathrm{yr}$ & none & 52.3 & $0.638[0.611,0.664]$ & 7.6 & 0.69 \\
\hline Nelson & age & $3 \mathrm{mo}$ & $12 \mathrm{yr}$ & none & 72.2 & $0.642[0.623,0.661]$ & 6.1 & 0.76 \\
\hline Shann & age & $1 \mathrm{yr}$ & none & none & 90.4 & $0.425[0.413,0.437]$ & 11.8 & 0.73 \\
\hline Theron & age & $1 \mathrm{yr}$ & $10 \mathrm{yr}$ & none & 52.3 & $0.960[0.921,0.999]$ & 3.1 & 0.70 \\
\hline Traub-Johnson & age $\&$ TBL & $1 \mathrm{yr}$ & $18 \mathrm{yr}$ & none & 90.4 & $0.703[0.687,0.719]$ & 7.1 & 0.81 \\
\hline Traub-Kichen & age \& TBL & $1 \mathrm{yr}$ & $17 \mathrm{yr}$ & $>74 \mathrm{~cm}$ & 90.1 & $0.629[0.614,0.643]$ & 8.4 & 0.81 \\
\hline
\end{tabular}

APLS- Advanced Pediatric Life Support, ARC- Australian Resuscitation Council, TBL- total body length, MUAC- mid-upper arm circumference, HL- humeral length

a percentage of the 1,938 children in the validation set for whom a weight would be predicted using this method 

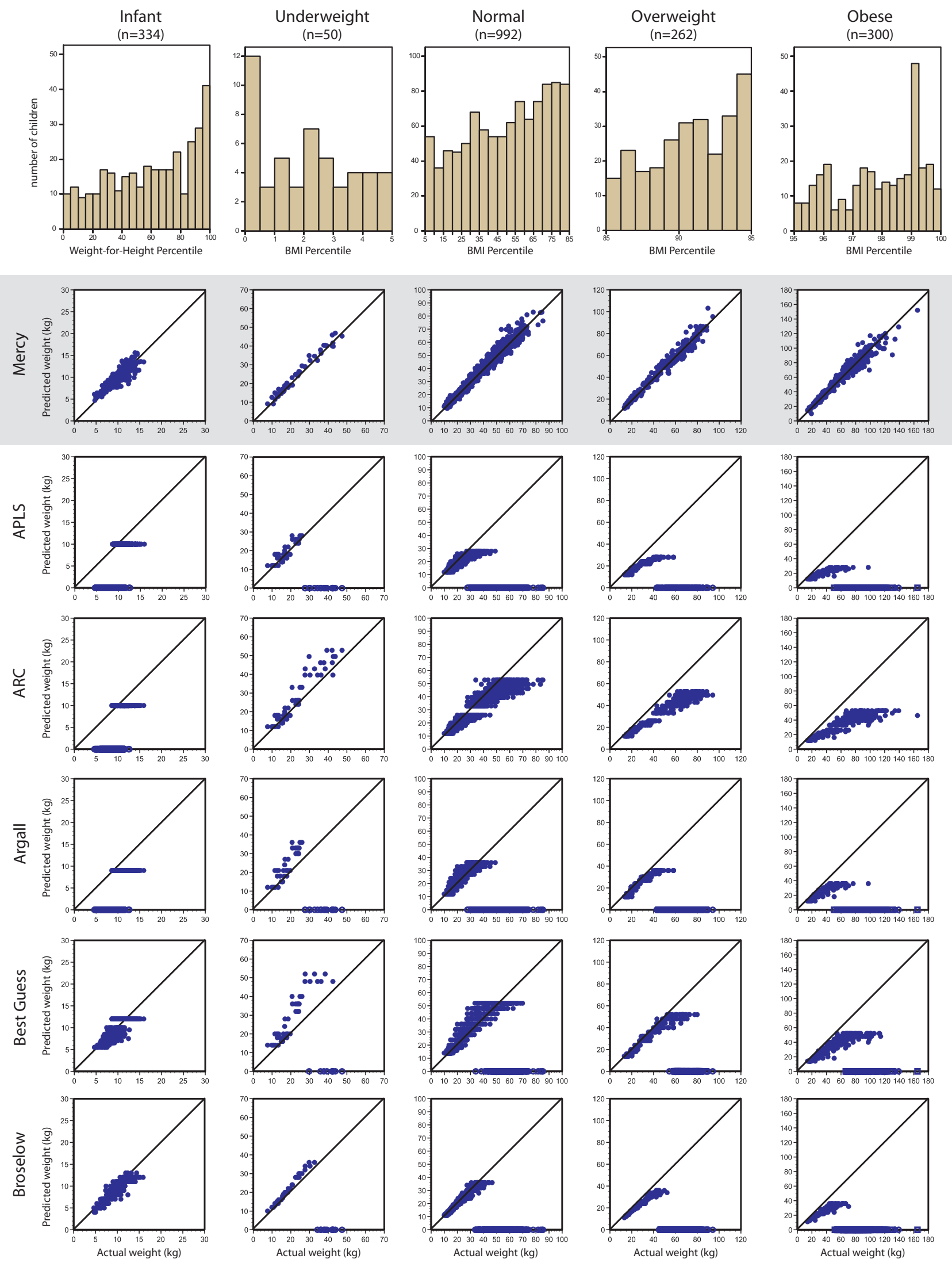

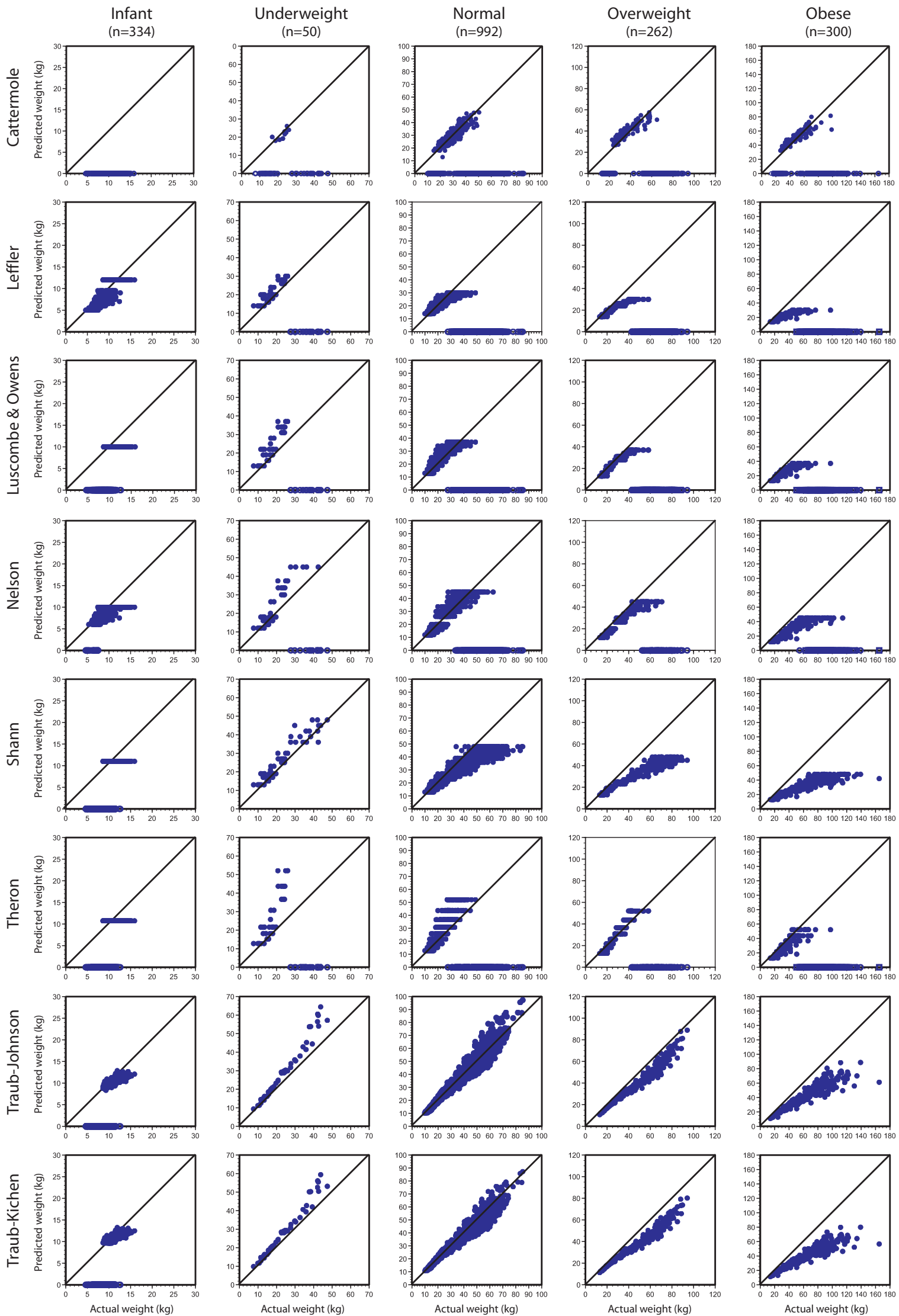

Fig. (3). Comparative performance of the Mercy Method. Histograms segregating the 1,938 children in the validation data set by weight category presented along with actual versus predicted weights by weight category for various weight estimation methods. Values that fall on the $\mathrm{x}$-axis indicate individuals for whom no weight could be calculated because length and/or age exceeded the bounds of the estimation method. 

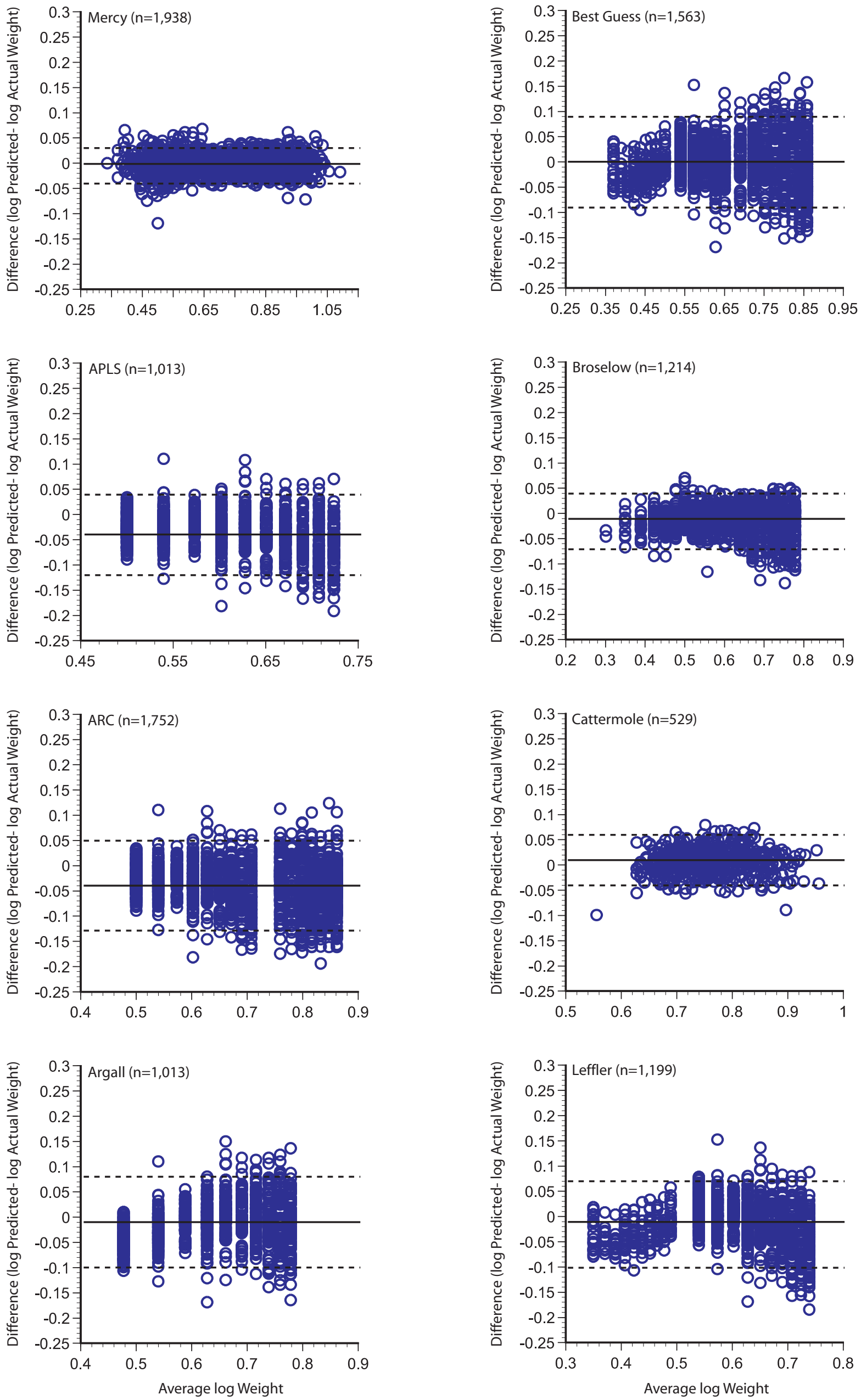

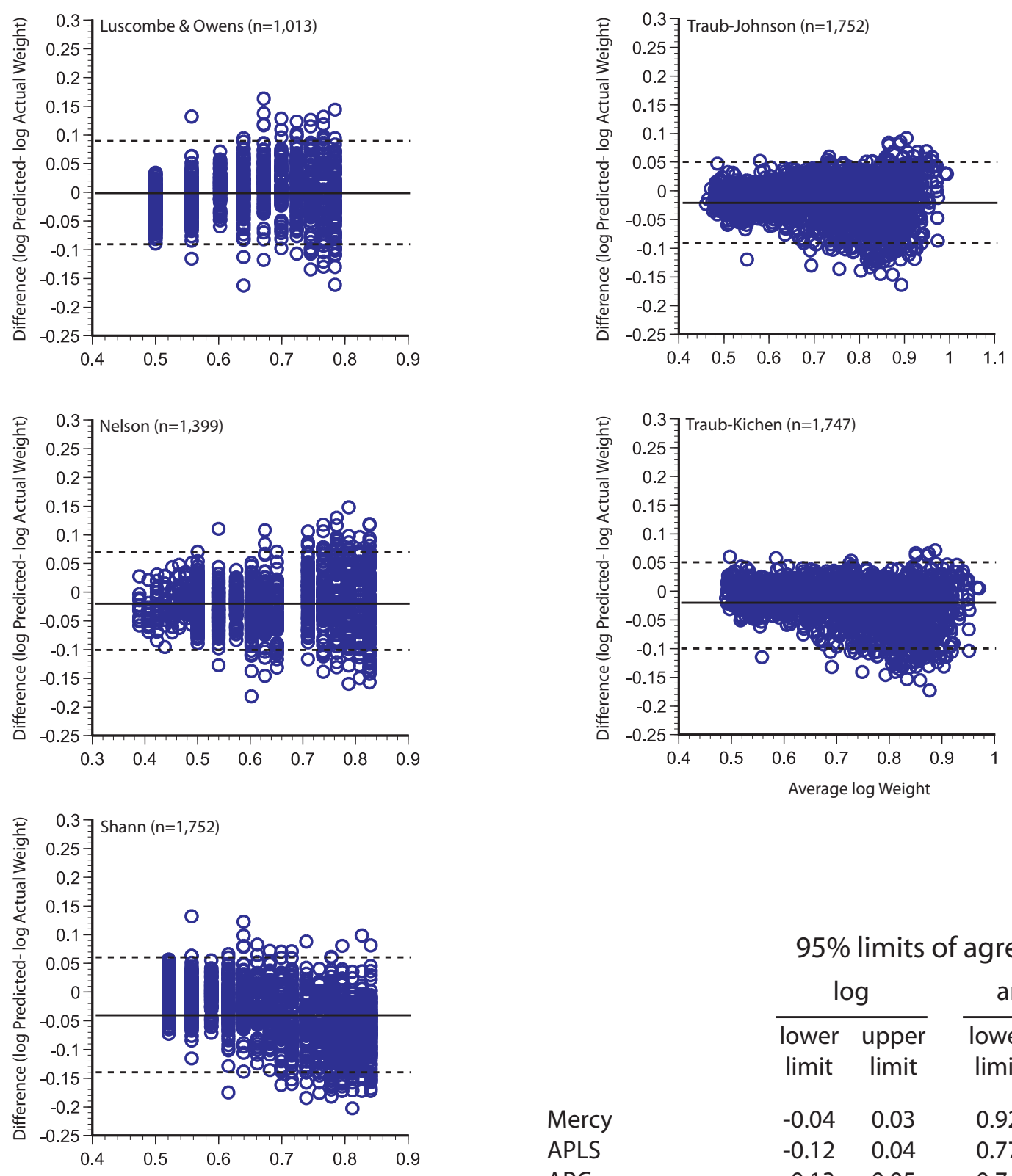

\begin{tabular}{|c|c|c|c|c|}
\hline & \multicolumn{4}{|c|}{$95 \%$ limits of agreement } \\
\hline & \multicolumn{2}{|c|}{$\log$} & \multicolumn{2}{|c|}{ antilog } \\
\hline & $\begin{array}{l}\text { lower } \\
\text { limit }\end{array}$ & $\begin{array}{c}\text { upper } \\
\text { limit }\end{array}$ & $\begin{array}{l}\text { lower } \\
\text { limit }\end{array}$ & $\begin{array}{l}\text { upper } \\
\text { limit }\end{array}$ \\
\hline Mercy & -0.04 & 0.03 & 0.92 & 1.08 \\
\hline APLS & -0.12 & 0.04 & 0.77 & 1.11 \\
\hline ARC & -0.13 & 0.05 & 0.74 & 1.12 \\
\hline Argall & -0.10 & 0.08 & 0.79 & 1.19 \\
\hline Best Guess & -0.09 & 0.09 & 0.81 & 1.23 \\
\hline Broselow & -0.07 & 0.04 & 0.85 & 1.10 \\
\hline Cattermole & -0.04 & 0.06 & 0.90 & 1.14 \\
\hline Leffler & -0.10 & 0.07 & 0.79 & 1.18 \\
\hline Luscombe \& Owens & -0.09 & 0.09 & 0.82 & 1.22 \\
\hline Nelson & -0.10 & 0.07 & 0.79 & 1.16 \\
\hline Shann & -0.14 & 0.06 & 0.72 & 1.14 \\
\hline Theron & -0.09 & 0.12 & 0.82 & 1.32 \\
\hline Traub-Johnson & -0.10 & 0.05 & 0.82 & 1.13 \\
\hline Traub-Kichen & -0.10 & 0.05 & 0.80 & 1.12 \\
\hline
\end{tabular}

Fig. (4). Bland-Altman plots. Bland-Altman plots depicting the log-transformed difference between predicted weight and actual weight vs. average weight for the 1,938 children in the validation data set. Dashed lines depict the $95 \%$ limits of agreements. The embedded table provides the back transformed values for the $95 \%$ limits of agreements. 
Table 3. Predictive Performance of the Weight Estimation Methods Evaluated in this Study

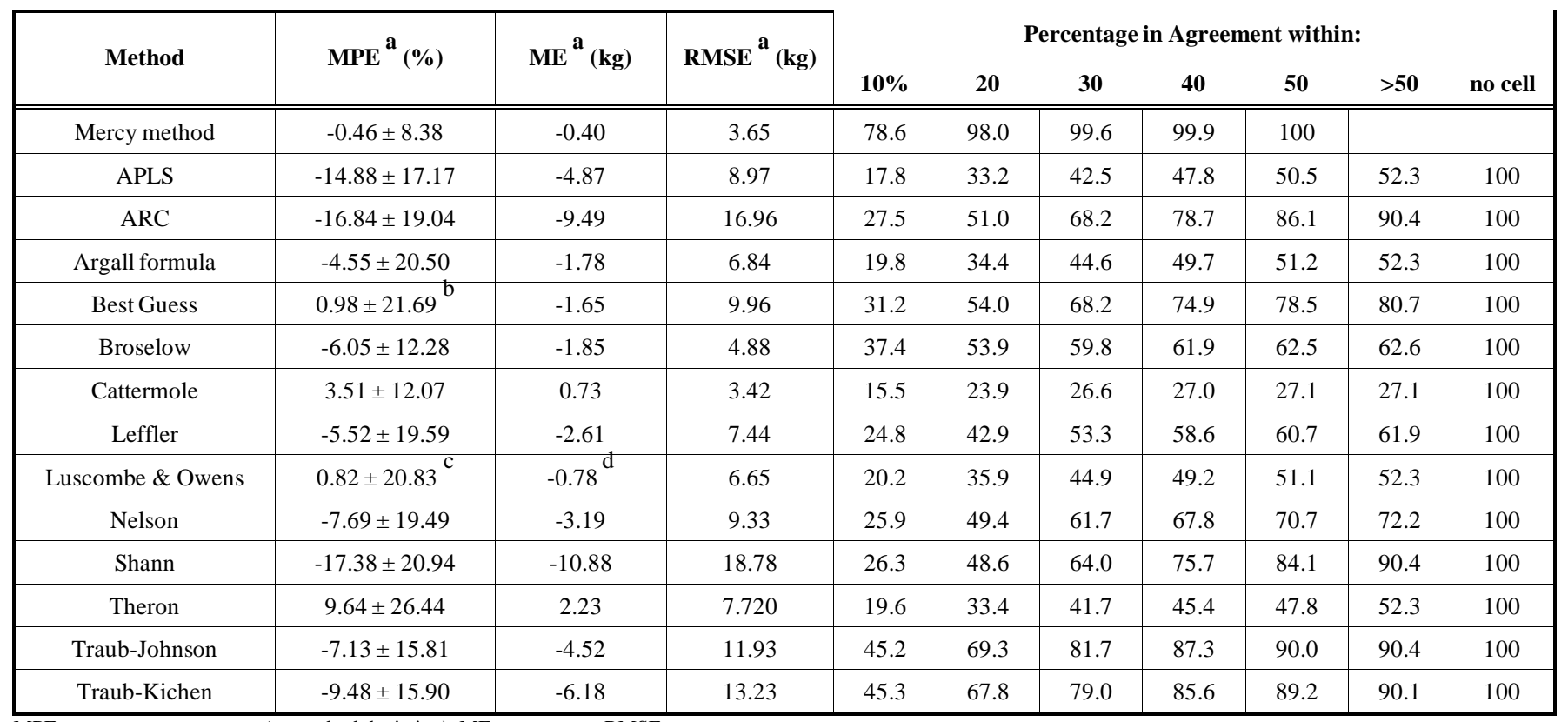

MPE- mean percentage error ( \pm standard deviation), ME, mean error, RMSE- root mean square error

a $\mathrm{p}<0.001$ between Mercy and all other methods except where designated, $\mathrm{b} p=0.03$ vs. Mercy, c $\mathrm{p}=0.04$ vs. Mercy, $\mathrm{d} p=0.002$ vs. Mercy

Table 4. Predictive Performance Of the Mercy Method Overall and by Demographic Subgroup

\begin{tabular}{|c|c|c|c|c|c|c|c|c|}
\hline \multirow{2}{*}{$\begin{array}{c}\text { Method } \\
\text { (n) }\end{array}$} & \multirow{2}{*}{$\begin{array}{c}\text { MPE } \\
(\%)\end{array}$} & \multirow{2}{*}{$\begin{array}{l}\text { ME } \\
(\mathbf{k g})\end{array}$} & \multirow{2}{*}{$\begin{array}{c}\text { RMSE } \\
(\mathbf{k g})\end{array}$} & \multicolumn{5}{|c|}{ Percentage in Agreement within: } \\
\hline & & & & $10 \%$ & 20 & 30 & 40 & $\mathbf{5 0}$ \\
\hline Mercy method (1938) & $-0.46 \pm 8.38$ & -0.40 & 3.65 & 78.6 & 98.0 & 99.6 & 99.9 & 100 \\
\hline male (1015) & $-0.49 \pm 8.50$ & -0.36 & 3.79 & 77.3 & 97.8 & 99.6 & 100 & \\
\hline female (923) & $-0.43 \pm 8.25$ & -0.46 & 3.50 & 80.0 & 98.3 & 99.7 & 99.9 & 100 \\
\hline Hispanic (742) & $-1.64 \pm 8.20$ & -0.86 & 3.89 & 77.8 & 98.4 & 99.7 & 100 & \\
\hline White (578) & $0.14 \pm 7.91$ & -0.17 & 3.20 & 80.1 & 98.4 & 100 & & \\
\hline Black (517) & $0.14 \pm 8.87$ & -0.14 & 3.77 & 78.3 & 97.3 & 99.4 & 99.8 & 100 \\
\hline Other (101) & $1.61 \pm 8.71$ & 0.27 & 6.54 & 77.2 & 97.0 & 98.0 & 100 & \\
\hline
\end{tabular}

MPE- mean percentage error ( \pm standard deviation), ME, mean error, RMSE- root mean square error

and precision, the Mercy method proved to be superior to other weight estimation strategies that were evaluated (Table 3). Our method demonstrated a significantly lower MPE, ME and RMSE than any of the other methods $(\mathrm{p}<0.05)$. Further, only the Mercy method estimated weights within $10 \%$ of actual for the majority of children in the validation set $(79 \%$ vs. 16 to $45 \%$ ). The performance criteria we observed for the previously published methods were consistent with those reported for these same methods in other validation and comparison driven investigations [7].

Given that previously published methods tend to perform poorly at the extremes of weight, the validation datasets were segregated by body-mass index (BMI) percentile and the Mercy method compared with the other weight estimation strategies by subgroup (note: infants under the age of 2 years are not routinely stratified into weight classes). As depicted in Fig. (3), the Mercy method performs robustly with minimal bias across the spectrum of ages and weight classes observed in this study. Table $\mathbf{4}$ details the performance characteristics of the Mercy Method by demographic subgroup.
Only slight variations in the Method's accuracy were observed by gender and race/ethnicity.

\section{DISCUSSION}

Weight estimation tools address a critical medical need in both developing and developed countries. They permit the determination of accurate weight-based doses in situations where there is neither the time nor the opportunity to directly weigh patients on a calibrated scale. Numerous weight estimation strategies have been described with each used to varying degrees in clinical practice. Though many have distinct advantages (e.g. simple age-based equations can be used without the need for reference materials, preprinted tables/tools limit the risk of calculation errors) most have significant limitations. Relatively few methods have been evaluated in multiple different pediatric populations and essentially no single previous method provides accurate estimates of weight across broad age- and weight-bands.

Most of the age-and length-based strategies examined in this study overestimated weight in children classified, by 
BMI, as underweight and underestimated weight in children classified as overweight or obese (Fig. 3). The degree to which this occurred depended largely on the constants driving their mathematical equations, with some methods biased toward more accurate prediction in children of lower weight (e.g. Broselow) and others performing better among children in the higher weight brackets (e.g. Theron). Irrespective of directionality, the bias observed with some methods in children at the extremes of weight represented as much as a 3fold error between predicted and actual weights. Discrepancies of this magnitude can be dangerous, and potentially lifethreatening, depending on how 'forgiving' the intervention or treatment that is being administered.

The singular habitus-based method (i.e. Cattermole) ranked among the best with respect to absolute bias and precision irrespective of BMI percentile. This is consistent with the finding that the relationship between weight and MUAC tends to be linear within any given population. Accordingly, MUAC-based methods should perform well in relatively homogenous populations although a greater degree of variance should be expected when compared with a method that incorporates both stature and girth. Given the nature of the data used to develop and validate the Mercy method, we could not compare the performance of our method to the Devised Weight Estimation Method (DWEM), the only other method to incorporate both body length and body habitus. Notably, the latter estimate involves a subjective rating of "slim," "average," or "heavy" [8]. While DWEM has been demonstrated to outperform age-based methods, the categorical assignment of habitus coupled with inconsistencies in subjective assessment between and within observers [interrater agreement- $78 \%$ (range: 58-93\%); intra-rater agreement- $86 \%$ (range: $81-94 \%$ )] contributed to bias and precision estimates that were larger than observed with strategies based on length [23].

The method we developed attempts to address several limitations inherent in the existing weight estimation strategies. As with other strategies, the Mercy method incorporates growth velocity but uses humeral length as a surrogate for total body length. Restricting length measurements to a single long bone minimize the discrepancies that result from whether the measurement is obtained with the child standing or lying down and may be easier and faster to obtain than total body length in a child who is uncooperative or obtunded. The Mercy method also incorporates habitus as a continuous variable which improves the accuracy of the overall length-based weight estimate and removes the subjective nature of categorizing the child's body type into one of a few alternatives. By developing a model with these considerations in mind we were able to expand the age range to which our weight estimation method can be applied and remove length restrictions which are typically imposed because of the disproportionate increase in weight-for-height observed as children get older. The combination of HL and MUAC as the length and habitus surrogates of choice for this investigation arise from the natural relationship between these two measurements, namely that MUAC is determined at the midpoint of the humerus and access to a single long bone can quickly yield both measurements. Ostensibly, methods designed around different long bone (e.g. ulna, tibia) and girth estimates (e.g. mid-thigh, abdomen), should demonstrate accuracy comparable to that of the Mercy Method provided that the measurements highly correlate with total body length and weight, respectively.

Limitations nested within this study need to be discussed principal among which is the fact that the NHANES data were collected by numerous raters not including the authors. As such, there is no mechanism to assess inter-rater reliability with respect to the precision of MUAC and HL measurements. In addition, the data span a collection range of 10 years which could fail to account for changing height-forweight patterns over time. Nonetheless, the Mercy Method performed robustly irrespective of these limitations. Finally, the NHANES data may not represent a truly random sample of children across race, ethnicity, socioeconomic status, etc. As such, our ability to extrapolate the suitability of this method to U.S. subgroups that are under sampled by NHANES along with non-U.S. populations is limited at this time.

The Mercy method was designed for use in the development of a measuring device (i.e. the Mercy TAPE) that performs assessments of length and girth simultaneously. The device can be printed on any flexible, non-stretchable medium (e.g. paper, plastic coated paper, fiberglass) so as to be disposable or semi-permanent. In its numeric form, the Mercy TAPE would have limited utility in settings where care providers are illiterate or do not use a written language. However, the tool could be easily revised with colors and/or symbols whose combination would correspond to a given dose, intervention strategy or weight target. While the Mercy method performs well in U.S. children, performance of the device is currently under evaluation.

\section{CONCLUSIONS}

The ideal weight estimation strategy should perform robustly across a broad range of ages and weights in all ethnic groups. This study demonstrates that the Mercy method satisfies the characteristics of a robust weight estimation strategy across the ages and weights encompassing the majority of pediatric patients. The results of ongoing external validation studies will further delineate the role of the Mercy method and the Mercy TAPE in children of varying ethnic and geographic origin.

\section{CONFLICT OF INTEREST}

The authors confirm that this article content has no conflicts of interest.

\section{ACKNOWLEDGEMENTS}

The authors gratefully acknowledge Dr. Stephen O'Neil for his critical review of the manuscript, Ms. Ashley Sherman for her assistance in downloading the NHANES data, and Mr. Barry Preuett for capturing the photographs.

\section{ABBREVIATIONS}

$\begin{aligned} \text { NHANES }= & \text { National Health and Nutrition Examination } \\ & \text { Survey, } \\ \text { MUAC }= & \text { mid- upper arm circumference, }\end{aligned}$




$\begin{array}{ll}\mathrm{HL} & =\text { humeral length, } \\ \mathrm{HHL} & =\text { half humeral length, } \\ \mathrm{CI} & =\text { confidence interval, } \\ \mathrm{ME} & =\text { mean error, } \\ \mathrm{MPE} & =\text { mean percentage error, } \\ \text { RMSE } & \text { root mean square error } \\ \text { REFERENCES }\end{array}$

[1] Taylor WR, Terlouw DJ, Olliaro NJ, Brasseur P, ter Kuile FO. Use of weight-for-age-data to optimize tablet strength and dosing regimens for a new fixed-dose artesunateamodiaquine combination for treating falciparum malaria. Bull World Health Organ 2006; 84: 956-64.

[2] Abuya TO, Molynuex CS, Orago ASS, Were S, Marsh V. Quality of care provided to febrile children presenting in rural private clinics on the Kenyan coast. Afr Health Sci 2004; 4: 160-70.

[3] Leffler S, Hayes M. Analysis of Parental Estimates of Children's Weights in the ED. Ann Emerg Med 1997, 30: 167-70.

[4] Krieser D, Nguyen K, Kerr D, Jolley D, Clooney M, Kelly AM. Parental weight estimation of their child's weight is more accurate than other weight estimation methods for determining children's weight in an emergency department? Emerg Med J 2007; 24: 7569.

[5] Goldman RD, Buskin S, Augartan A. Parental estimate of their child's weight: accurate for resuscitation drug doses. Pediatr Emerg Care 1999; 15: 19-21.

[6] Nieman CT, Manacci CF, Super DM, Mancuso C, Fallon WF Jr. Use of the Broselow tape may result in the underresuscitation of children. Acad Emerg Med 2006; 13: 1011-9.

[7] World Health Organization. Technical consultation on the use of pharmacokinetic analyses for paediatric medicine development. Geneva, Switzerland. 2009.

[8] Garland JS, Kishaba RG, Nelson DB, Losek JD, Sobocinski KA. A rapid and accurate method for estimating body weight. Am J Emerg Med 1986; 4: 390-3.

[9] Traub SL, Kichen L. Estimating ideal body mass in children. Am J Hosp Pharm 1983; 40: 107-10.
[10] Tinning K, Acworth J. Make your best guess: an updated method for paediatric weight estimation. Emerg Med Australas 2007; 19: 535-41.

[11] Needlman RD. The First Year. In: Behrman RE, Kliegman RM, Jenson HB, Eds. Nelson Textbook of Pediatrics. $17^{\text {th }}$ ed. Philadelphia: Saunders 2004; p. 31.

[12] Oakley PA. Inaccuracy and delay in decision making in paediatric resuscitation, and a proposed reference chart to reduce error. BMJ 1988; 297: 817-9.

[13] Centers for Disease Control and Prevention (CDC). National Center for Health Statistics (NCHS). National Health and Nutrition Examination Survey Data. Hyattsville, MD: U.S. Department of Health and Human Services, Centers for Disease Control and Prevention, 1999-2008. Available at: http://www.cdc.gov/nchs/nhanes/nha-nes_questionnaires.htm.

[14] Mackway-Jones K, Molyneux E, Phillips B, Wieteska S. Advanced Paediatric Life Support. London: BMJ Books 2001.

[15] The Advanced Life Support Committee of the Australian Resuscitation Council. Paediatric advanced life support - the Australian resuscitation council guidelines. Med J Aust 1996; 165: 199-206.

[16] Argall JA, Wright N, Mackway-Jones K, Jackson R. A comparison of two commonly used methods of weight estimation. Arch Dis Child 2003; 88: 789-90.

[17] Lubitz D, Seidal JS, Chameides L, Luten RC, Zaritsky AL, Campbell FW. A rapid method for estimating weight and resuscitation drug dosages from length in the pediatric age group. Ann Emerg Med 1988, 17: 576-81.

[18] Cattermole GN, Leung PYM, Maka PSK, Graham CA, Rainer TH. Mid-arm circumference can be used to estimate children's weights Resuscitation 2010; 81: 1105-10.

[19] Luscombe M, Owens B. Weight estimation in resuscitation: is the correct formula still valid? Arch Dis Child 2007; 92: 412-5.

[20] Hazell W. Emergency medicine at your fingertips, $2^{\text {nd }}$ ed. New Zealand: MediMedia (NZ) 2000; pp. 132. 15

[21] Theron L, Adams A, Jansen K, Robinson E. Emergency weight estimation in Pacific Island and Maori children who are large-forage. Emerg Med Australas 2005; 17: 238-43.

[22] Traub SL, Johnson CE. Comparison of methods of estimating creatinine clearance in children. Am J Hosp Pharm 1980; 37: 195201.

[23] Black K, Barnett P, Wolfe R, Young S. Are methods used to estimate weight in children accurate? Emerg Med 2002; 14: 160-5.

Received: June 26, 2012

(C) Abdel-Rahman and Ridge; Licensee Bentham Open.

This is an open access article licensed under the terms of the Creative Commons Attribution Non-Commercial License (http://creativecommons.org/licenses/by-nc/3.0/) which permits unrestricted, non-commercial use, distribution and reproduction in any medium, provided the work is properly cited. 